\title{
Ministry of Health Saudi Arabia: COVID-19 Website Benefits and Validity and Reliability of the Data
}

\author{
Hossam Alakhrass' ${ }^{(1)}$, Abdullah Al Mulla2 ${ }^{2}$, Sameh Kamal Al-Akhrass ${ }^{3}$, Mohammed Aljuaid ${ }^{4}$ \\ ${ }^{1}$ Department of Public Health, Maternity and Children's Hospital, Ministry of Health, Dammam, Saudi Arabia \\ ${ }^{2}$ Radiology Department, Dammam, Ministry of Health, Dammam, Saudi Arabia \\ ${ }^{3}$ Department of Occupational Health, Ministry of Health, Dammam, Saudi Arabia \\ ${ }^{4}$ Department of Health Administration, King Saud University, Riyadh, Saudi Arabia \\ Email: drhossamk@hotmail.com, aamulla@moh.gov.sa, sal-akhrass@moh.gov.sa,maljuaid@ksu.edu.sa
}

How to cite this paper: Alakhrass, $\mathrm{H} ., \mathrm{Al}$ Mulla, A., Al-Akhrass, S.K. and Aljuaid, M. (2021) Ministry of Health Saudi Arabia: COVID-19 Website Benefits and Validity and Reliability of the Data. Health, 13, 4046.

https://doi.org/10.4236/health.2021.131004

Received: July 3, 2020

Accepted: January 19, 2021

Published: January 22, 2021

Copyright $\odot 2021$ by author(s) and Scientific Research Publishing Inc. This work is licensed under the Creative Commons Attribution International License (CC BY 4.0).

http://creativecommons.org/licenses/by/4.0/

\begin{abstract}
Since the declaration of the Covid-19 pandemic by the World Health Organization on March 11, 2020, the coronavirus has upended communities and dramatically changed people's daily endeavors across the globe [1]. The current Covid-19 circumstances are occasioned by social realities such as the proliferation of misinformation, social inequalities, and nations' inability to prepare for unprecedented crises such as the coronavirus. While Covid-19 has managed to elicit anxiety, cause illnesses and deaths, different sectors, individuals, and health dockets utilize technology to combat the pandemic. As we advance, there will be a need for a critical social understanding of how technology can be used to apprehend global crises such as coronavirus. To comprehend how technology is being used to address the coronavirus crises, the current paper assesses, evaluates, and rates the Covid-19 website developed by the Ministry of Health of Saudi Arabia [2].
\end{abstract}

\section{Keywords}

Covid-19, Coronavirus, Saudi Arabia, MOH, Validity, Reliability

\section{Introduction}

As Covid-19 continues to wreak havoc across the globe, the government of Saudi Arabia recognizes the pandemic as a serious humanitarian, health and development crisis. Part of the strategies to share data about the nation's progress in handling corona virus was the development of a Covid-19 website

https://covid19.moh.gov.sa/. The website has been of great aid in briefing the citizens about the condition of Covid-19 in Saudi Arabia. 


\subsection{User Benefits}

\section{Learning/awareness}

The Covid-19 website by the Ministry of Health (MOH) Saudi Arabia is a crucial platform for sharing information about coronavirus as a pandemic. Since coronavirus is a new disease there is a need for enhanced awareness. The website is accessible to all citizens connected to the internet. Covid-19 has affected all facets of life, including social, economic, and political spheres. However, a significant majority of citizens lack the real facts and data on the exact impacts [3]. The Covid-19 website by $\mathrm{MoH}$ Saudi Arabia comes in handy to bridge this gap. The awareness will go a long way in helping the citizens cope with the pandemic and plan for the uncertainties occasioned to their businesses, jobs, etc.

Since Covid-19 is a unique disease, there exists numerous misinformation and myths in the public domain [4]. The website comes in handy to provide necessary information about COVID 19 disease, its symptoms, mode of transmission, and treatments available in the Kingdom. Visually appealing charts and explanatory videos provide understandable and straightforward instructions for the public. Preventive methods are described using comprehensive charts. Instructions for washing hands, home quarantine, personal protective equipment, and multiple another day-to-day situation handling are explained very well. Instructions cover all range of populations, from the most vulnerable group to the ordinary citizens.

The website forms a viable platform for the government to appeal to all citizens to battle the virus. The government expects the residents to promptly follow approved guidelines, instructions, and warnings, create caution, and avoid panic [1]. The website also stipulates all the world health organization's policies include observing physical distancing at all possible circumstances, quarantine measures, whenever applicable, use of personal protective equipment like face masks, gloves, etc. The government aims to spread deep awareness of the pandemic's impact and make its residents equipped to face any emergency; in this ultra-modern era of information technology, the Covid-19 website is the mostsuitable channel to achieve this goal [1] [2].

\subsection{Monitoring Trends}

Numerous computer-based approaches are used to present Covid-19 data. The approaches are specifically intended to help people identify and monitor trends of Covid-19. The government can quickly inform its citizens on the daily confirmed cases, deaths, and recoveries via the website. The information will help people plan. For instance, if the number of confirmed cases is flattening, the government and the people can quickly start reopening and returning to their daily normal. Simultaneously, increment in the number of Covid-19 positive cases will also help citizens adjust and prepare for future consequences [1] [2]. The website also stipulates all the world health organization's policies include observing physical distancing at all possible circumstances, quarantine measures, 
whenever applicable, use of personal protective equipment like face masks, gloves, etc.

The Covid-19 pandemic has proven to be unpredictable across the world [5]. The same situation is taking place in Saudi Arabia. The website can automatically generate trends crucial to understanding the coronavirus dynamics concerning the social, economic, and political aspects of life.

The process of monitoring trends is instrumental in dealing with a pandemic such as Covid-19. The extent of Covid-19 can be best understood within the context of daily number of confirmed cases. This is an important role that the website continues to fulfil even at a point when corona virus is still causing havoc across the globe Saudi Arabia included.

\section{Validity and Reliability of the Data}

The data is transferred directly from the ministry of health's database to the Covid-19 website without altering any figures. Cumulative information is depicted as an alternate tab that provides the nation's complete Covid-19 history to date. This tab provides information based on cases per different cities, totally confirmed and active cases (Graph 1), totally recovered cases (Graph 2), real tests performed, and overall deaths (Graph 3 ). The colour coding and numerical representation provide an easy understanding of data. The presentation of data on the map helps quick identification of confirmed and recovered cases [1]. Notes and definition sections are presented at the right bottom of the "cumulative" page, which allows the common man to understand the terms used, data sources, and how data is derived. This section also details how deaths are recorded in Covid-19 related death certificates. The graphical representation includes total cases presented separately on a logarithmic scale and a normal scale. The validity and consistent reliability of the data fulfil the mandate of the Covid-19 website by MoH Saudi Arabia to provide accurate and verifiable coronavirus information.

\section{Presentation of Data}

The presentation of data largely determines how the process of interpretation is undertaken. The Covid-19 website by MoH Saudi Arabia provides a clear visual presentation of the covid-19 data based on the following variables:

* Number of confirmed cases

Total confirmed cases have been crucial in comprehending the extent to which Covid-19 has been impacting in different regions. The Covid-19 website by MoH Saudi Arabia provides a simple graph of confirmed cases on a daily basis. As evident below, the chart captures different states and regions and one can interpret the information presented easily. This form of delivering data is crucial since it meets the motif of the website which is to inform the public in the simplest way possible. Data for all variables are also presented in the Graphs 1-4 presented. 


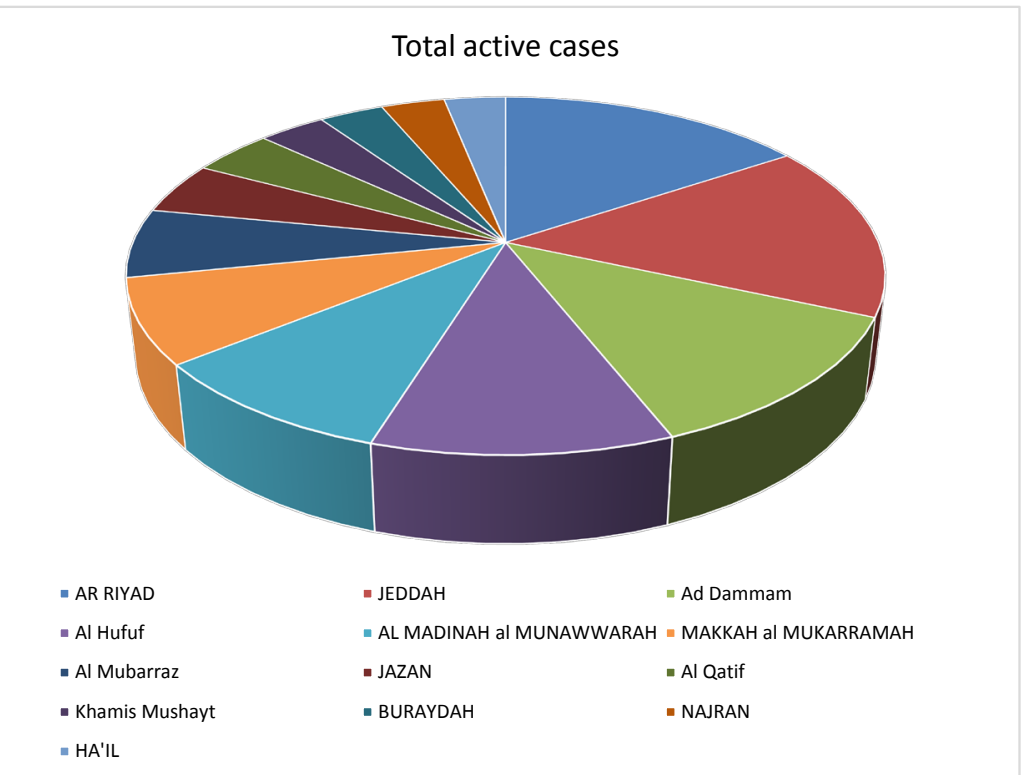

Graph 1. Number of confirmed cases.

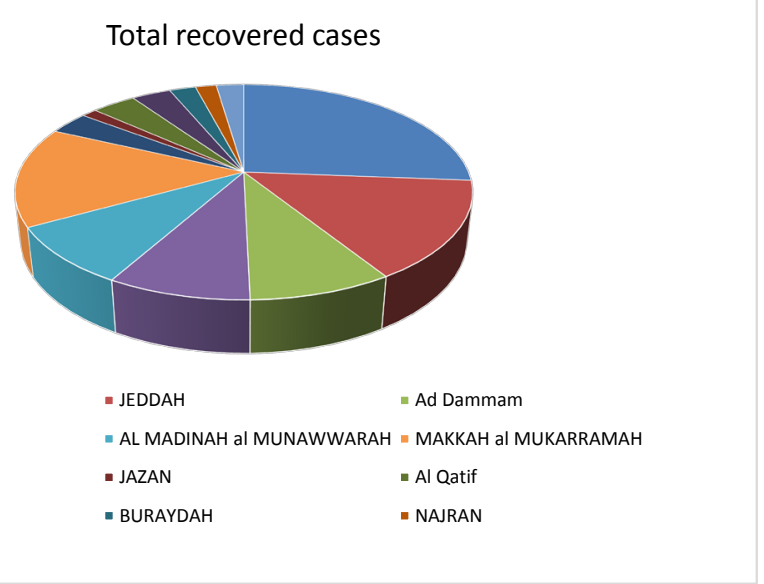

Graph 2. Number of recoveries.

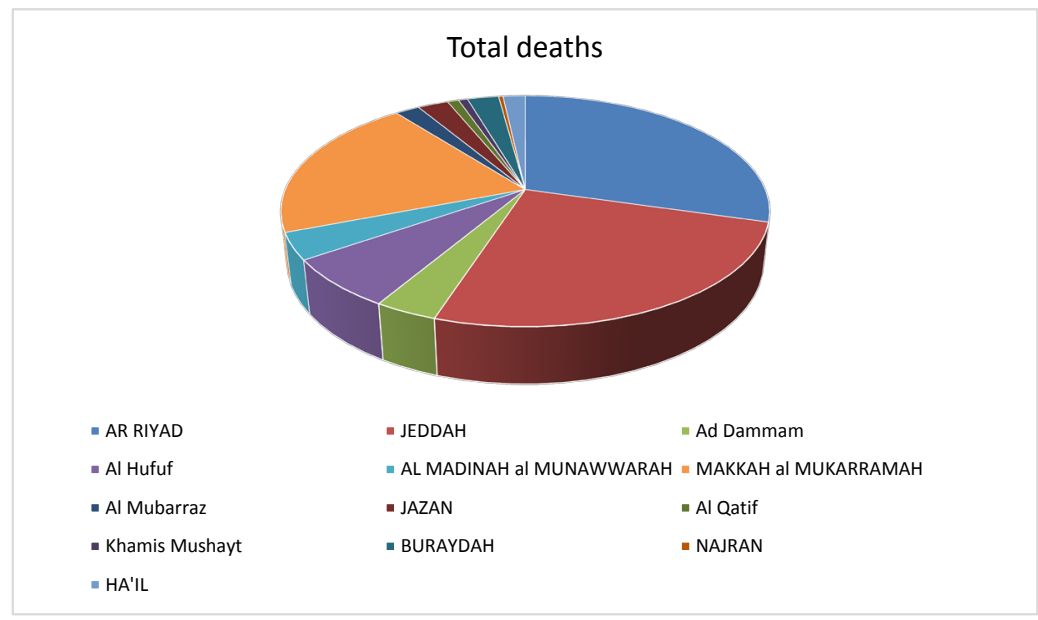

Graph 3. Number of deaths. 


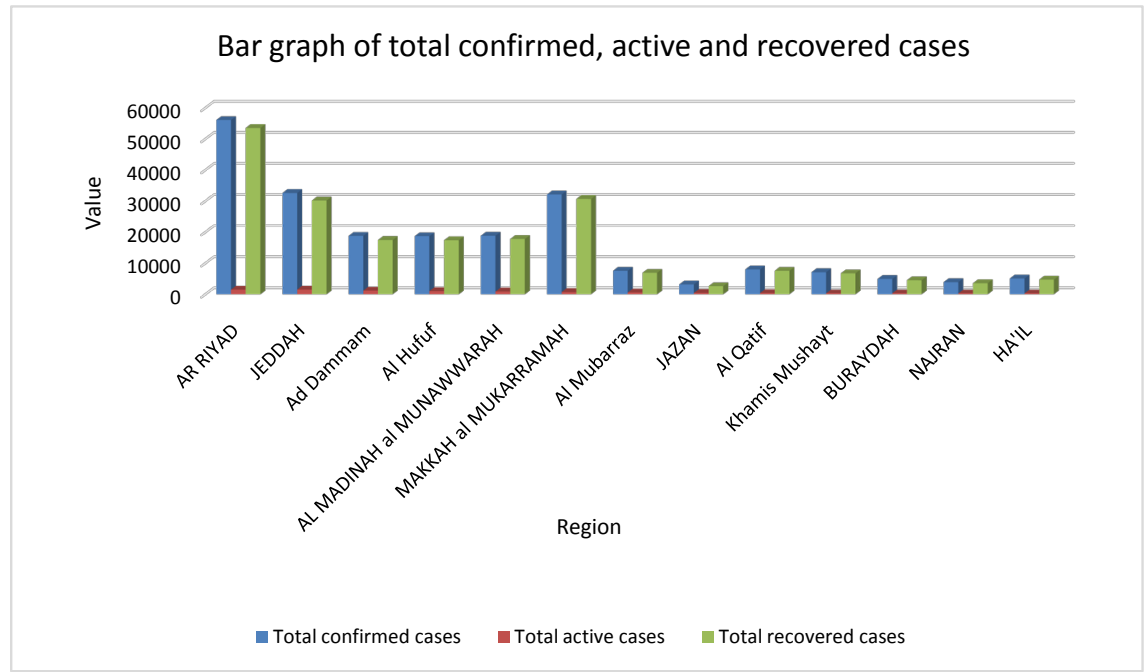

Graph 4. Geographical region.

Therefore, it is easy to scan the data from a glimpse and acquire the exact information that one wants. The dashboard summarizes vital information that would help an individual translate the data trends on covid-19 in Saudi Arabia in an accessible manner. The variables mentioned present the most demanded details by all citizens. The website considers the pertinent aspects that members of the public need to know at every given point. People have been overly concerned with the number of cases reported, how many people have died, and which region has the highest number of cases. With a simple presentation, this information on the dashboard, the website has managed to play its role in disseminating critical data to the intended audience.

\section{Accessibility}

One of the critical ingredients of a viable website is ease of access. The Covid-19 website by MoH Saudi Arabia offers all citizens an easy and equal opportunity for access. Since the website's launch, there have been minimal instances of downtimes, and therefore, the site can be accessed on a 24/7 basis. The website also implements audio features for people with disabilities such as the blind. As such, people with disabilities can also acquire every detail about Covid-19 by a click of a button. The website does not require any exceptional internet connectivity to access. Further, The Covid-19 website by MoH Saudi Arabia is compatible with all the browsers, which guarantees all users ease of access.

The user interface is simple and easy to manoeuvre across all the data. The technical aspects have been simplified to allow every individual irrespective of their computer literacy skills, to access the website.

\section{Key Concepts}

Confirmed case: This is a suspected case which has been ascertained through a laboratory test. 
Recovered case: A previously conformed case which no longer reveals Covid-19 infection.

Symptomatic patient: A Covid-19 individual who reveals outright symptoms such as fever, headaches, etc.

Asymptomatic patient: a Covid-19 positive case but does not reveal direct signs and symptoms of coronavirus.

Active cases: Total deaths, recovered cases, and confirmed cases.

The website defines all the technical terms in a bid to help the audience understand different aspects of coronavirus. The website does not in any way assume that citizens comprehend all the terms about Covid-19. As such, the definition of the tern's section is critical to helping everyone understand the dynamics of coronavirus.

\section{Conclusion}

Overall, Covid-19 is a crucial precursor to panic, depression, and stigma, which emanates from misinformation. Since the emergence of the Covid-19 pandemic, the evolving nature of the virus, its symptoms, medication, and its spread are significant concerns for nations. However, countries such as Saudi Arabia have initiated web-based solutions to communicate with the masses. The Covid-19 website by MoH Saudi Arabia provides abrupt and all-time valid and reliable information about the coronavirus. This information is playing a pivotal role in eradicating the virus. Evidently, the virus can only be contained if people acquire accurate and relevant information consistently. It is no doubt that technology necessitates effective communication, and the website has managed to disseminate data about Covid-19 to all people in real-time.

\section{Conflicts of Interest}

The authors declare no conflicts of interest regarding the publication of this paper.

\section{References}

[1] Jebril, N. (2020) World Health Organization Declared a Pandemic Public Health Menace: A Systematic Review of the Coronavirus Disease 2019 “COVID-19”. Available at SSRN 3566298. https://doi.org/10.2139/ssrn.3566298

[2] COVID 19 Dashboard: Saudi Arabia. (n.d.). Retrieved October 30, 2020. https://covid19.moh.gov.sa/

[3] Shah, S.G.S. and Farrow, A. (2020) A Commentary on "World Health Organization Declares Global Emergency: A Review of the 2019 Novel Coronavirus (COVID-19)". International Journal of Surgery, 76, 128-129. https://doi.org/10.1016/j.ijsu.2020.03.001

[4] Ohannessian, R., Duong, T.A. and Odone, A. (2020) Global Telemedicine Implementation and Integration within Health Systems to Fight the COVID-19 Pandemic: A Call to Action. JMIR Public Health and Surveillance, 6, e18810. https://doi.org/10.2196/18810 
[5] Alkhamees, A.A., Alrashed, S.A., Alzunaydi, A.A., Almohimeed, A.S. and Aljohani, M.S. (2020) The Psychological Impact of COVID-19 Pandemic on the General Population of Saudi Arabia. Comprehensive Psychiatry, 102, e152192.

https://doi.org/10.1016/j.comppsych.2020.152192 